\title{
The Effect of Intravenous Prednisolone Treatment on the Retinal Nerve Fiber Layer in Multiple Sclerosis Patients with Optic Neuritis
}

\author{
Hande Husniye Telek, ${ }^{1}$ Ahmet Kaderli, ${ }^{1}$ Ozgur Balta, ${ }^{1}$ Gulten Sungur, ${ }^{1}$ Mehmet Yakın, ${ }^{1}$ Dicle Hazırolan, ${ }^{1}$ \\ Evin Singar Ozdemir, ${ }^{1}$ Ugur Emrah Altıparmak, ${ }^{2}$ Firdevs Ornek ${ }^{1}$
}

1Department of Ophthalmology, Ankara Training and Researching Hospital, Ankara, Turkey

${ }^{2}$ Department of Ophthalmology, Acıbadem University Faculty of Medicine, Ankara, Turkey

\begin{abstract}
Objectives: The aim of this study was to investigate the potential impact of intravenous (IV) prednisolone treatment on the retinal nerve fiber layer (RNFL) in multiple sclerosis (MS) patients with optic neuritis (ON).

Methods: The demographic features, systemic illnesses, laboratory results, ophthalmological examination findings and RNFL measurements of 28 MS patients who had IV prednisolone treatment for ON and of 24 MS patients with ON that was not treated were retrieved and reviewed retrospectively.

Results: Of the 52 patients included in the study, 31 were women and 21 were men. The average age in the prednisolone group was $32 \pm 7.15$ years (range: $28-40$ years) and it was $34 \pm .2$ years (range: $30-42$ years) in patients who did not receive treatment. The initial RNFL in the treatment group was $116.8 \mathrm{I} \pm 5.4 \mathrm{I} \mu \mathrm{m}$ and it was $78.13 \pm 3.94 \mu \mathrm{m}$ at the third month, while it was I 18.2 $\pm 6.07 \mu \mathrm{m}$ initially and $80.09 \pm 5$. I $8 \mu \mathrm{m}$ at the third month in the untreated group. There was no statistically significant difference between the groups in the RNLF evaluation ( $p>0.05)$

Conclusion: Treatment with IV prednisolone had no distinct effect on RNFL.

Keywords: Multiple sclerosis, optic neuritis, prednisolone, retinal nerve fiber layer.
\end{abstract}

\section{Introduction}

Optic neuritis (ON) is inflammation that damages the optic nerve and is characterized by a sudden onset of vision loss and dyschromatopsia. Visual symptoms typically progress for a few weeks and then begin to improve on their own within the first month. $\mathrm{ON}$ is often associated with existing or future multiple sclerosis (MS), and is frequently the first symptom. According to data provided by the Optic Neuritis Study Group (ONSG), the 10-year risk of developing MS after an episode of ON was $38 \%$, and $56 \%$ in those with I or more demyelinating lesions observed with magnetic res- onance imaging (I).

MS is a demyelinating disease in which inflammation affects the central nervous system. It most frequently develops in young adults. In a histopathological review of MS patients, partially preserved axons, numerous demyelinated areas, and loss of oligodendrocyte and astroglial scarring were observed (2). Thinning of nerve fibers without myelin was significant in identifying the axonal loss.

The retinal nerve fiber layer (RNFL) comprises nonmyelinated retinal ganglion cell axons and is the most proximal region of the afferent visual pathway. Changes in the thickness of the RNLF reflect alteration in the axonal integrity of

Address for correspondence: Hande Husniye Telek, MD. Sukriye Mahallesi, Ulucanlar Caddesi, Ankara Egitim ve Arastırma Hastanesi Ankara, Turkey

Phone: +90 3125953467 E-mail: handetelek@gmail.com

Submitted Date: July 18, 2017 Accepted Date: October 04, 2017 Available Online Date: December 04, 2017

${ }^{\circ}$ Copyright 2017 by Beyoglu Eye Training and Research Hospital - Available online at www.beyoglueye.com 
the optic nerve. Retrobulbar damage due to axonal degeneration is identified by optic nerve pallor and RNFL defect (3).

Optical Coherence Tomography (OCT) is now used in a variety of neuro-ophthalmic cases. Among them are anterior ischemic optic neuropathy, toxic and other inflammatory optic neuropathies, MS, optic-spinal MS, pseudotumor cerebri, migraine, optic nerve head drusen, Leber's hereditary optic neuropathy, Alzheimer's disease, and Friedrich's ataxia (4). In studies conducted with MS patients, there was demonstrated RNFL thinning. Such variations in the RNFL were correlated with the activity of the disease and the size of the demyelinating plate identified in MRG $(4,5)$.

OCT is a noninvasive and easy-to-use medical imaging system which provides a cross-sectional image of the retina using the reflection and absorption of light waves (6). It can provide a high resolution assessment of the anatomical structure of the retina, and is also used in the examination of the optic nerve and peripapillary RSLT.

More than $50 \%$ of MS patients have $\mathrm{ON}$ at some point (7). Thinning of the RNFL has also been demonstrated in MS patients who have never had ON $(7,8)$. The purpose of this study was to assess the impact of IV methylprednisolone treatment on the thickness of RNFL in MS patients.

\section{Methods}

The files of patients diagnosed with $\mathrm{ON}$ in January 2013 through May 2015 were reviewed and examined retrospectively. Approval of the study protocol was obtained from the institutional review board. The study and data collection protocols complied with all local laws and the principles of the Declaration of Helsinki.

A total of 52 eyes of 52 patients complying with the study criteria were included in the study. The patients were divided into 2 groups: those with $\mathrm{ON}$ that was treated and those with $\mathrm{ON}$ that was not treated. Of the 28 patients incorporated in the treatment group, 17 were women $(61 \%)$ and II were men (39\%). Of the 24 patients who were not treated, I 4 were women $(58 \%)$ and 10 were men $(42 \%)$.

The treatment protocol was $250 \mathrm{mg}$ intravenous (IV) methylprednisolone; I mg/kg/day oral prednisone was administered to the patients in the treatment group for 3 days, 4 times a day. The non-treatment patients were those who refused the treatment or had vital contraindications for treatment.

\section{Inclusion Criteria}

- Those who were clinically and definitively diagnosed with MS,

- Those with a visual acuity level that was suitable for OCT measurement, and

- Those who had suffered an attack of $O N$ at least 3 months previously.

\section{Exclusion Criteria}

- History of glaucoma or with intraocular pressure (IOP) exceeding $21 \mathrm{mmHg}$,

- Existence of ocular trauma or history of other ocular surgery,

- Diabetic retinopathy identified by ophthalmoscopy,

- History of neurological disease,

- Existence of accompanying ocular disease (glaucoma, uveitis, etc.),

- Explicit refractive defect (a spherical defect of more than 4 diopters or an astigmatic defect of more than 3 diopters),

- Any vascular and autoimmune disease (diabetes mellitus, hypertension, Buerger's disease, vasculitis, etc.), or

- Opacities impeding retinal tomographic imaging.

A definitive clinical and laboratory diagnosis was made based on patient files. All demographic data, records of systemic disease present, initial ophthalmological examination results, and OCT results from both eyes were recorded.

All participants underwent full ophthalmological examination. Reactive defects were measured using an automated refractometer. The best corrected visual acuity was identified for each eye using a Snellen chart. The anterior segment was evaluated through biomicroscopic examination. IOP was measured and indirect ophthalmoscopy examination was conducted with non-contact fundus lens (SuperField; Volk Optical, Inc., Mentor, OH, USA) after having achieved mydriasis.

RNFL thickness analysis was performed using a Stratus OCT device, version 6.0.2 (Carl Zeiss Meditec AG, Jena, Germany). The fast RNFL thickness protocol of 3 consecutive $360^{\circ}$ scans with a diameter of $3.4 \mathrm{~mm}$ centered on the optic disc was performed. The OCT cross-section was fixed such that the center of the measurement circle was the center of the optic disc. RNFL results were recorded in the temporal, superior, and inferior quadrants. The video was monitored during the measurement and the imaging was repeated if required, in order to maintain the fixation point.

SPSS for Windows, Version 15.0 software (SPSS, Inc., Chicago, IL, USA) was used for the statistical analysis. An independent samples t-test was used for the comparison of measurements between groups. Comparisons within each group were conducted using a paired t-test. Statistical significance was determined to be $p<0.05$.

\section{Results}

The mean age of the 52 patients recruited for the study was $35 \pm 6.4$ years (range: $28-42$ years); the mean age was $32 \pm 7.15$ years (range: $28-40$ years) in the treatment group and $34 \pm 8.2$ years (range: $30-42$ years) in the non-treatment group. The age difference between the 2 groups was not statistically significant $(p>0.05)$. 
Table I. Retina nerve fiber layer thickness measurements

\begin{tabular}{|c|c|c|c|c|c|c|}
\hline & $\begin{array}{c}\text { Untreated group } \\
\text { Initial measurement }\end{array}$ & $\begin{array}{c}\text { Treated group } \\
\text { Initial measurement }\end{array}$ & $\mathbf{P}$ & $\begin{array}{l}\text { Untreated group } \\
\text { 3rd month }\end{array}$ & $\begin{array}{c}\text { Treated group } \\
\text { 3rd month }\end{array}$ & $\mathbf{P}$ \\
\hline Mean RNFL & $118.12 \pm 6.07$ & || $6.8| \pm 5.4|$ & $>0.05$ & $80.09 \pm 5.18$ & $78.13 \pm 3.94$ & $>0.05$ \\
\hline Superior RNFL & $133.89 \pm 5.02$ & $130.12 \pm 7.72$ & $>0.05$ & $116.15 \pm 3.73$ & $113.12 \pm 2.05$ & $>0.05$ \\
\hline Nasal RNFL & $89.15 \pm 7.08$ & $87.31 \pm 6.96$ & $>0.05$ & $72.52 \pm 4.94$ & $71.44 \pm 7.06$ & $>0.05$ \\
\hline
\end{tabular}

RNFL: Retinal nerve fiber layer.

In all, 3 I of the patients were women (59.6\%) and 2 I were men (40.4\%); 17 of the 22 patients were women $(61 \%)$ and II were men (39\%) and I 4 of 24 patients in the treatment group were women $(58 \%)$ and 10 were men (42\%). The gender difference between the 2 groups was not statistically significant $(p>0.05)$.

Initially, the mean RNFL thickness was $\mid \mathrm{I} 6.8 \mathrm{I} \pm 5.4 \mathrm{I} \mu \mathrm{m}$ in the treated group and $118.12 \pm 6.07 \mu \mathrm{m}$ in the untreated group. The RNFL in the treated group was $\mathrm{I} .3 \mathrm{I} \mu \mathrm{m}$ thinner than that of the other group; however, the difference was not statistically significant $(p>0.05)$. The difference between the RNFL measurements of the 2 groups conducted in 4 quadrants was not statistically significant (Table I).

At the third month, the mean RNFL thickness was $78.13 \pm 3.94 \mu \mathrm{m}$ in the treated group and $80.09 \pm 5.18 \mu \mathrm{m}$ in the untreated group. The difference of $1.86 \mu \mathrm{m}$ was not statistically significant $(p>0.05)$. The difference between the groups in RNFL thickness conducted in 4 quadrants was not statistically significant (Table I).

Superior quadrant RNFL thickness had decreased by $17.00 \mu \mathrm{m}$ in the treated group and $17.74 \mu \mathrm{m}$ in the untreated group at the third month compared to the initial measurement; however, the difference between the 2 groups was not statistically significant ( $p>0.05)$. In the inferior quadrant, RNFL thickness had decreased by $13.54 \mu \mathrm{m}$ in the treated group and by $17.06 \mu \mathrm{m}$ in the untreated group after 3 months when compared with the initial measurement. The difference between the groups was not statistically significant ( $p>0.05)$. RNFL thickness in the temporal quadrant had decreased by $20.90 \mu \mathrm{m}$ in the treated group and by 21.84 $\mu \mathrm{m}$ in the untreated group at the third month. The differ-

Table 2. Variation in retinal nerve fiber layer thickness in the treated and untreated groups

\begin{tabular}{lccc} 
RNFL & Treated group & Untreated group & P \\
\hline Initial RNFL & $116.8 I \pm 5.4 \mathrm{I}$ & $1 \mathrm{I} 8.12 \pm 6.07$ & $>0.05$ \\
3rd month RNFL & $78.13 \pm 3.94$ & $80.09 \pm 5.18$ & $>0.05$ \\
\hline
\end{tabular}

RNFL: Retinal nerve fiber layer. ence between the 2 groups was not statistically significant ( $p>0.05$ ). It was also seen that RNFL thickness of the nasal quadrant had decreased by $15.87 \mu \mathrm{m}$ in the treated group and by $16.63 \mu \mathrm{m}$ in the untreated group after 3 months. The decrease in $\mu \mathrm{m}$ was greater in the untreated group than in the treated group, but the difference between the groups was not statistically significant ( $p>0.05$ ) (Table I).

RNFL thickness was seen to have decreased in all quadrants in the untreated group at the third month compared with the original measurements. The difference between the 2 groups was statistically significant $(p<0.05)$. The comparison of all quadrants with each other yielded the observation that RNFL thickness, especially in the temporal quadrant, was thinner at the end of 3 months and this result was statistically significant $(p<0.05)$. The comparison of thinning in other quadrants in the untreated group did not generate statistically significant results $(p>0.05)$ (Table I).

RNFL thickness had also decreased in all quadrants in the treated group at the third month compared with the initial measurements. The difference between the groups was statistically significant $(p<0.05)$. The comparison of all quadrants revealed that RNFL thickness, particularly in the temporal quadrant, was thinner at the end of 3 months and that the difference was statistically significant $(p<0.05)$. The comparison of thinning in other quadrants in the treated group did not generate statistically significant results $(p>0.05)$ (Table 2$)$.

\section{Discussion}

MS is a demyelinating disease of the central nervous system, the etiology of which is not properly known. Various factors are thought to be responsible for demyelination, and different neurological and systemic symptoms arise as a result of demyelination. Among them are motor deficits, followed by sensorial complaints, and finally, visual impairment. In general, it is a syndrome of multifocal disorders where gliosis is observed with histological demyelination and inflammatory variations in a series of relapses and remissions (5).

Following an episode of $\mathrm{ON}$, inflammation of the optic 
nerve causes axonal degeneration and optic nerve atrophy. In various studies, treatment of I g/day of IV methylprednisolone has been applied during an attack of $\mathrm{ON}$ to reduce visual loss; however few have assessed the impact of IV methylprednisolone upon RNFL thickness.

Corticosteroids are the initial episode treatment option in cases of ON (9). However, the appropriate dosage is still controversial (I0). The use of oral prednisolone as a monotherapy is ineffective and may increase the risk of further attacks (II). ONSG data indicated that the patient group medicated first with $250 \mathrm{mg}$ IV methylprednisolone 4 times for 3 days followed by I mg/kg/day oral prednisolone reached definitive visual acuity in shorter period than a placebo group (I). In a study conducted by Yahu et al. (I2) on 20 patients diagnosed with $\mathrm{ON}$, it was demonstrated that II patients medicated with IV methylprednisolone recovered their visual acuity in an average 13 days, while 9 patients not treated recovered their visual acuity in 18 days. Akyurekli et al. (13) studied 55 patients diagnosed with acute $\mathrm{ON}$ and found that 39 patients medicated with IV methylprednisolone improved in II days, while I 6 patients not treated improved in 4 I days. In our study, we observed that visual acuity improved 3 lines in patients treated with IV methylprednisolone in 7 days and in 3 days in the untreated group.

Studies of retinal neurodegeneration in MS have a long history and there has been an increase in the number of such studies in recent years. Voght (I4) first demonstrated that there was a clear loss in the retinal nerve fiber layer during his assessment of patients with a history of $\mathrm{ON}$ using direct ophthalmoscopy and a green filter in 1913 .

RNFL is largely made up of retinal ganglion cell axons. The optic nerves are also an extension of ganglion cells in the retina oriented toward the brain. In the retina, these axons are without myelin, but and they are covered by a myelin sheath formed by oligodendrocytes after exiting the retina. The retinal nerve fibers are the most proximal parts of the visual transmission pathways, and RNFL volume provides information about the axonal integrity of the optic nerve and its structure. That is, any variation in RNFL demonstrates axonal response.

Qualitative variations in RNFL in MS patients were defined for the first time by Frisen and Hoyt (15) in 1974. It was Parisi et al. (16), in 1999, who reported quantitative variations for the first time (16). That study was the first to use OCT in MS. OCT is a relatively new and practical method that allows for quantitative measurement of the retinal fiber layer in MS.

OCT provides cross-sectional imaging of biological tissue through high resolution. The axial resolution of OCT is 8 to 10 microns. It is possible to obtain cross-sectional images similar to those observed under a microscope. The degree of visualization that can be achieved with OCT is sufficient for use as a noninvasive tissue biopsy. OCT not only provides imaging of the optic disc in the retina and anatomical structures such as the macula, but also allows for the examination of intra-retinal structures, such as the retinal nerve fibers, photoreceptors, and retina pigment epithelium. Furthermore, the quantitative retinal measurements obtained provide valuable information for the diagnosis and follow-up of disease. RNFL thickness measured by OCT serves as a good indicator of axonal degeneration and may constitute a good model for neurobiological studies of MS.

Parisi et al. (16) studied RNFL thickness in I4 MS patient I year after an $\mathrm{ON}$ attack and the results were compared the measurements of 14 healthy controls. The mean RNFL thickness in the eyes of the control group was II I.I I $\pm 1 \mathrm{I} .42$ $\mu \mathrm{m}$, while it was $82.73 \pm 10.73 \mu \mathrm{m}$ in the unaffected eyes of the patients and $59.79 \pm 10.80 \mu \mathrm{m}$ in the affected eyes. The RSLT thickness in the eyes that had an ON attack was significantly lower than that of the other eye or the control group. The examination of differences between quadrants demonstrated that RNFL thinning was more distinct in the temporal quadrant. The mean RNFL thickness in the unaffected eyes were thinner than that seen in the control group, but did not reach a level of significance. In the same study, the measurements of RNFL thickness were compared with visual acuity and it was observed that RNFL thinning remained despite the fact that visual function fully recovered following the ON attack. They reported $46 \%$ thinning in the affected eyes and $28 \%$ in the unaffected eyes.

Fisher et al. (17) also found that RNFL thickness was distinctly lower in the ON eyes of 90 MS patients compared with 36 healthy controls. The authors demonstrated that there was significant RNFL thinning in the ON eyes of asymptomatic MS patients. Costello et al. (6) conducted the first prospective study. For a period of I year, every 3 months they observed 54 patients who had an ON attack. RNFL thickness was lower in the ON eyes, as seen in the other studies, and no difference was observed between quadrants. A decrease in RNFL thickness was found in $11 \%$ of the patients at $3 \mathrm{rd}$ month following the attack, $85 \%$ of the patients in the 6 th month, and $4 \%$ of the patients at the 12 th month. The temporal area was the quadrant where thinning was most frequently observed to occur $(16,18,22)$. The loss of NFL thickness may be stabilized 7 to 12 months following the attack (19).

In our study, as in the literature, the examinations of variations between quadrants demonstrated that the most frequently and most markedly affected quadrant was the temporal area.

Although OCT is a strong imaging device in the examination of optic nerve and peripapillary RNFL, it has also been demonstrated that there are significant differences between 
devices in the RSLT thickness results obtained as a consequence of different methods of data analysis employed (20). Due to such issues, the RNFL thickness of all patients in this study was always measured using the same device.

In various studies, the authors demonstrated that RNFL thinning existed in the temporal quadrant following an attack of ON (6, 20, 21). Trip et al. (22) studied II patients with MS who had a single attack of $\mathrm{ON}$ and 14 patients who had a clinically isolated syndrome, and they found that RNFL thickness decreased despite an increase in visual acuity following ON. Yahu et al. (I2) also demonstrated that the loss of RNFL thickness was similar in patients both with and without IV methylprednisolone treatment, and that there was thinning in all quadrants, especially the temporal quadrant, at the third month in all patients. In our study, thinning in RNFL thickness was found at the third month following the attack in both the treated and the untreated group, and was greater in the temporal quadrant compared with the other quadrants. The degree of thinning in the treated patients was similar to that of the untreated patients; our results were consistent with those of Yahu et al.

The results provided here suggest that thinning in the temporal quadrant may be the initial result of axonal loss further to optic neuritis, variations in RNFL are not correlated with visual acuity, and that IV methylprednisolone treatment had no impact on variations in RNFL.

\section{Disclosures}

Peer-review: Externally peer-reviewed.

Conflict of Interest: None declared.

Authorship Contributions: Involved in design and conduct of the study (HHT, AK); preparation and review of the study (OB, GS); data collection (HHT, OB); and statistical analysis (AK).

\section{References}

I. Beck RW, Trobe JD, Moke PS, Gal RL, Xing D, Bhatti MT, et al. High- and low-risk profiles for the development of multiple sclerosis within 10 years after optic neuritis: experience of the optic neuritis treatment trial. Arch Ophthalmol 2003;121:944-9.

2. Lublin FD, Miller AE. Multiple sclerosis and other inflammatory demyelinating diseases of the central nervous system. In: Bradley WG, Daroff RB, Fenichel GM, Jankovic J, editors. Neurology in Clinical Practice. 5th ed. Philadelphia: Elseiver; 2008. p. |584-6|4.

3. Elbøl P, Work K. Retinal nerve fiber layer in multiple sclerosis. Acta Ophthalmol (Copenh) 1990;68:48I-6.

4. Mudun A. Optic Coherence Tomography in Neuro-Ophthalmological Diseases. Acıbadem University Health Sciences Magazine 20I I;2:5-9.

5. Current therapy in neurologic disease. In: Bakar M, çev ed. 7. baskı. Güneş Tıp Kitabevi; 2007. p. 185-208.

6. Costello F, Coupland S, Hodge W, Lorello GR, Koroluk J, Pan YI, et al. Quantifying axonal loss after optic neuritis with optical coherence tomography. Ann Neurol 2006;59:963-9.
7. Cheng H, Laron M, Schiffman JS, Tang RA, Frishman LJ. The relationship between visual field and retinal nerve fiber layer measurements in patients with multiple sclerosis. Invest Ophthalmol Vis Sci 2007;48:5798-805.

8. Pueyo V, Martin J, Fernandez J, Almarcegui C, Ara J, Egea C, et al. Axonal loss in the retinal nerve fiber layer in patients with multiple sclerosis. Mult Scler 2008;|4:609-14.

9. Van Stavern GP. Management of optic neuritis and multiple sclerosis. Curr Opin Ophthalmol 200I; 12:400-7.

10. Brusaferri F, Candelise L. Steroids for multiple sclerosis and optic neuritis: a meta-analysis of randomized controlled clinical trials. J Neurol 2000;247:435-42.

I I. Beck RW, Gal RL, Bhatti MT, Brodsky MC, Buckley EG, Chrousos GA, et al. Visual function more than 10 years after optic neuritis: experience of the optic neuritis treatment trial. Am J Ophthalmol 2004; 137:77-83.

12. Yau GS, Lee JW, Lau PP, Tam VT, Wong WW, Yuen CY. Prospective study on retinal nerve fibre layer thickness changes in isolated unilateral retrobulbar optic neuritis. ScientificWorldJournal 20I3;2013:6946I3.

13. Akyurekli O, Celebisoy N. Optic neuritis: Results with pulse methylprednisolone. J Neurol Sci Turk 2009;26:8-I I.

14. Vogt A. Herstellung eines gelbblauen Lichtfiltrates, in welchem die Makula centralis in vivo in gelber Farbe erscheint, die Nervenfasern der Netzhaut und andere feine Einzelheiten derselben sichtbar werden und der Grad der Gelbfärbung der Linse ophthalmoskopisch nachweisbar ist. Graefe's Arch Clin Exp Ophthalmol 1913;84:293-31।.

15. Frisén L, Hoyt WF. Insidious atrophy of retinal nerve fibers in multiple sclerosis. Funduscopic identification in patients with and without visual complaints. Arch Ophthalmol 1974;92:91-7.

16. Parisi V, Manni G, Spadaro M, Colacino G, Restuccia R, Marchi $S$, et al. Correlation between morphological and functional retinal impairment in multiple sclerosis patients. Invest Ophthalmol Vis Sci 1999;40:2520-7.

17. Fisher JB, Jacobs DA, Markowitz CE, Galetta SL, Volpe NJ, Nano-Schiavi ML, et al. Relation of visual function to retinal nerve fiber layer thickness in multiple sclerosis. Ophthalmology 2006; I 1 3:324-32.

18. Sepulcre J, Murie-Fernandez M, Salinas-Alaman A, García-Layana $A$, Bejarano B, Villoslada P. Diagnostic accuracy of retinal abnormalities in predicting disease activity in MS. Neurology 2007;68:|488-94.

19. Costello F, Hodge W, Pan YI, Eggenberger E, Coupland S, Kardon $\mathrm{RH}$. Tracking retinal nerve fiber layer loss after optic neuritis: a prospective study using optical coherence tomography. Mult Scler 2008; | 4:893-905.

20. Pro MJ, Pons ME, Liebmann JM, Ritch R, Zafar S, Lefton D, et al. Imaging of the optic disc and retinal nerve fiber layer in acute optic neuritis. J Neurol Sci 2006;250: I I4-9.

2I. Henderson AP, Trip SA, Schlottmann PG, Altmann DR, Garway-Heath DF, Plant GT, et al. An investigation of the retinal nerve fibre layer in progressive multiple sclerosis using optical coherence tomography. Brain 2008; |31:277-87.

22. Trip SA, Schlottmann PG, Jones SJ, Altmann DR, Garway-Heath DF, Thompson AJ, et al. Retinal nerve fiber layer axonal loss and visual dysfunction in optic neuritis. Ann Neurol 2005;58:383-91. 